Case report

\title{
Recurrence of Seminoma in the Scrotum after Orchidectomy
}

\author{
Yasuhiko Hirose, Hidetoshi Akita, Toshiki Kato, Hideyuki Kamisawa, \\ Yoshinobu Moritoki and Takehiko Okamura
}

Department of Nephro-Urology, Anjo Kousei Hospital, Aichi, Japan

\begin{abstract}
Recurrence of seminoma in the scrotum after inguinal orchidectomy is extremely rare. Herein, we report the second case in the literature of recurrence of seminoma in the scrotum following inguinal orchidectomy for stage I seminoma.
\end{abstract}

Key words: recurrence, seminoma, hydrocele

(J Rural Med 2008; 3(2): 34-36)

\section{Introduction}

Recurrence of seminoma in the scrotum following inguinal orchidectomy is an extremely rare disease. Only one case has previously been reported (by Carey et al.). We report the second local recurrence of seminoma in the scrotum in the literature.

\section{Case Report}

A 52-year-old man was presented to our hospital complaining of right painful scrotal swelling on February 10,2004. He first noticed the swelling at the beginning of 2001, but did not seek treatment until his right scrotum gradually became enlarged and painful.

Physical examination revealed a $90 \times 50 \mathrm{~mm}$ palpable hard mass with a hydrocele in the right scrotum. No superficial lymph nodes were palpable. Laboratory analysis included normal hematologic and blood chemical values. Creactive protein was $1.7 \mathrm{mg} / \mathrm{dl}$ (normal values: $<0.5$ ). Preoperative serum tumor markers showed an AFP level of 3.4 $\mathrm{ng} / \mathrm{ml}$ (normal values: <10.0), HCG- $\beta$ level of $0.2 \mathrm{ng} / \mathrm{ml}$ (normal values: $<0.1$ ) and LDH of $278 \mathrm{U} / 1$ (normal values: 119-229). A CT scan revealed a $50 \mathrm{~mm}$ irregular enhanced tumor with calcification and a hydrocele around it in the

Correspondence to: Yasuhiko Hirose

Department of Nephro-Urology, Anjo Kousei Hospital, 28

Higashihirokute, Anjo-cho, Anjo, Aichi 446-8602, Japan

E-mail: yh3@yahoo.co.jp right scrotum and no enlarged lymph node or metastasis (Fig. 1).

A standard right inguinal orchidectomy was performed on February 12, 2004. Histological examination revealed a pure seminoma with extensive invasion of the tunica albuginea (Fig. 2). There was no spermatic cord invasion. The liquid of the right hydrocele was yellowish and clear and amounted to $50 \mathrm{ml}$. The patient was diagnosed as having a stage I testicular seminoma and was treated without adjuvant therapy.

A routine follow-up CT scan 3 months after orchidectomy showed a $5 \mathrm{~mm}$ enhanced nodule. Since tumor markers including AFP, HCG- $\beta$ and LDH were normal after 3 months, we believed this to be a postoperative change. However, 3 months later, the patient found that the size of the nodule had increased and complained of intermittent pain in the nodule. Ultrasound, CT and MRI examinations

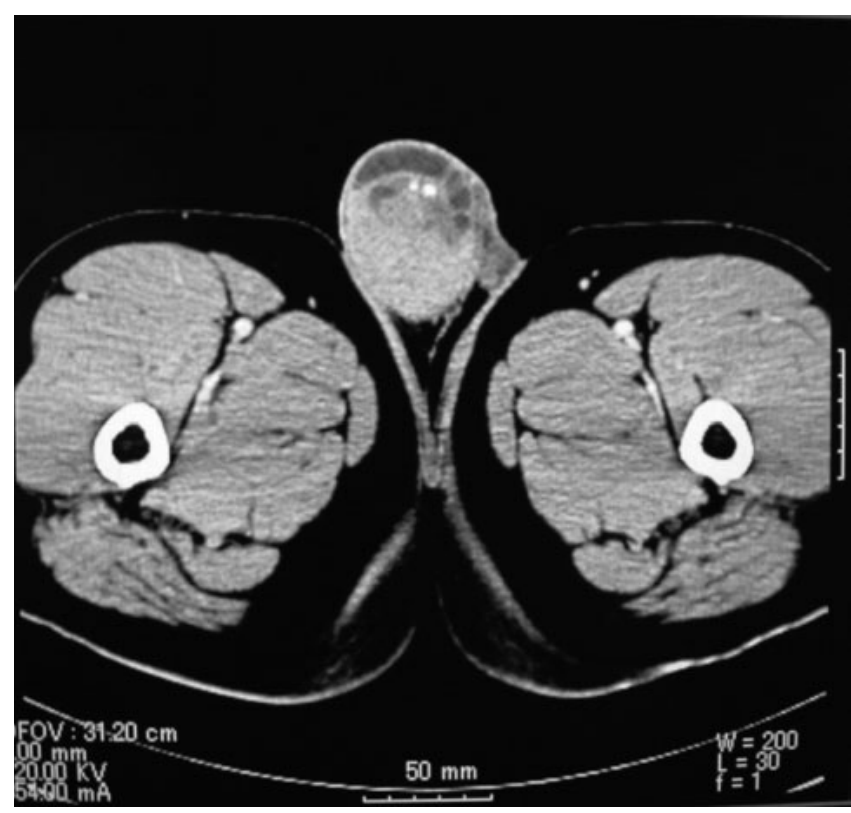

Figure 1 CT image showing a $50 \mathrm{~mm}$ irregular enhanced tumor with calcification and a hydrocele around it in the right scrotum. 


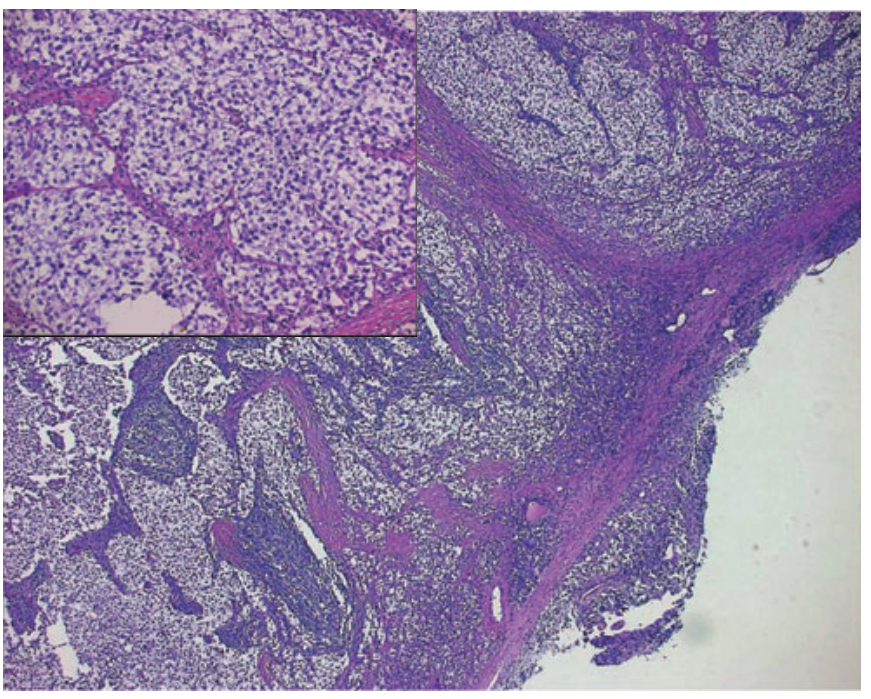

Figure 2 Pathological specimen of the right testicular tumor obtained from the first orchidectomy. The image shows a pure seminoma with extensive invasion of the tunica albuginea. (Hematoxylin and eosin stain, $\times 100$ )

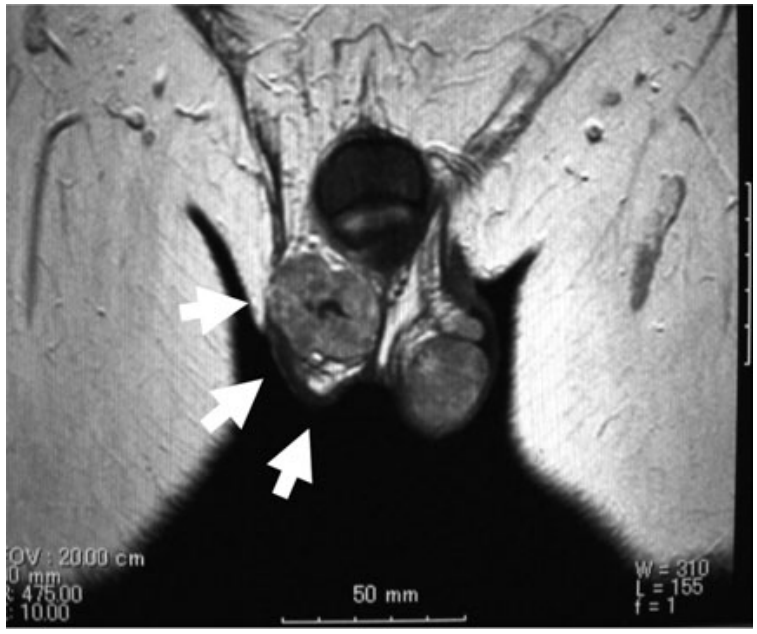

Figure 3 Coronal MRI image of the scrotum at the sight of local recurrence of testicular tumor. This postcontrast, T1-weighted image show a $30 \mathrm{~mm}$ tumor with diffuse enhancement. a)

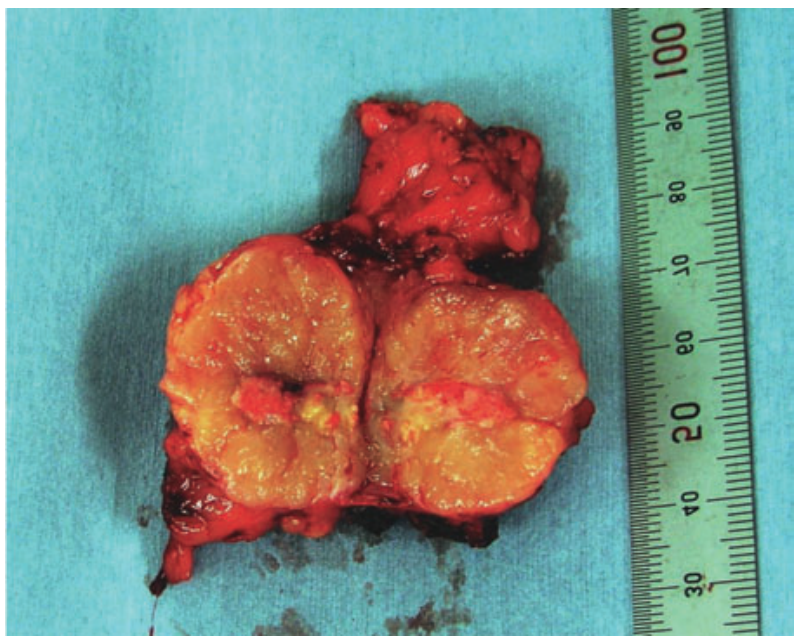

b)

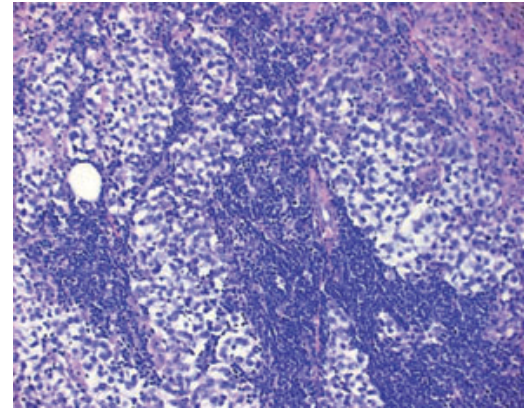

Figure 4 a) Macroscopically, the specimen appears to be a $30 \times 26 \times 20 \mathrm{~mm}$ yellow-white solid tumor. b) Microscopically, the specimen appears to be a recurrent seminoma. (Hematoxylin and eosin stain, $\times 400$ )

revealed a $30 \mathrm{~mm}$ nodule in the scrotum (Fig. 3).

We suspected that local recurrence of seminoma in the scrotum, and resection of the nodule was performed on December 17, 2004, 11 months after orchidectomy. Pathological examination confirmed recurrent seminoma. The surgical margin was histologically negative (Fig. 4).

Although we suggested adjuvant radiotherapy for the para-aortic/paracaval, ipsilateral iliac and inguinal lymph node yields and ipsilateral scrotum, he wanted adjuvant radiotherapy for only the ipsilateral scrotum. The daily dose was $2 \mathrm{~Gy}$ with linear accelerators. The total dose was $24 \mathrm{~Gy}$. He remains well with no evidence of disease 3 years later.

\section{Discussion}

Seminomas account for approximately $40 \%$ of all testicular tumors, and the majority of patients are diagnosed in the early stages of the disease. About $15 \%$ to $20 \%$ of all seminomas metastasize, usually to the para-aortic lymph nodes. Metastasis or recurrence in the scrotum in cases operated on by inguinal orchidectomy is extremely rare.

Capelouto et al. reported that local recurrence of testicular tumor following uncomplicated trans/inguinal orchidectomy occurred in $4(0.4 \%)$ out of 976 patients, and local recurrence of a preoperative stage I testicular tumor following uncomplicated trans/inguinal orchidectomy occurred in $1(0.1 \%)$ out of 707 patients. They also reported no local recurrence of seminoma following uncomplicated 
trans/inguinal orchidectomy in any of the 469 patients in their study ${ }^{1)}$.

Carey et al. reported the first case of local recurrence of seminoma following uncomplicated trans/inguinal orchidectomy ${ }^{2}$. Our case is the second case in the literature. In the present case, pathological examination at orchidectomy revealed a seminoma with invasion of the tunica albuginea and an accompanying right hydrocele. Although hydrocele cytology was not performed, it might had been positive. In a prior report of positive hydrocele cytology accompanying seminoma, Clyde et al. proposed two contributory mechanisms: (1) metastatic lymphatic obstruction with retrograde flow into the subserosal tunica vaginalis, with subsequent tumor shedding into the hydrocele fluid, and (2) direct tumor extension through the tunica albuginea and visceral tunica vaginalis ${ }^{3)}$. When the orchidectomy was performed, the hydrocele was not ruptured in any obvious way; however, malignant cells may have disseminated during the operation, and this may have resulted in local recurrence.

The disease-specific survival for stage I seminoma is approximately $100 \%$, irrespective of the choice of adjuvant treatment. However, the prognosis of seminoma with local recurrence remains unclear.

\section{References}

1. Capelouto CC, Peter EC, Bernard JR. A Review of scrotal violation in testicular cancer: is adjuvant local therapy necessary?. J Urol 1995; 153: 981-985.

2. Carey BM, Jones WG, Robinson RG. Case report: seminoma metastatic to the scrotum. Clin Radio 1997; 52: 240-241.

3. Clyde RR, Norman EP. Positive hydrocele cytology accompanying testis seminoma. Urol 1992; 19: 292-293. 